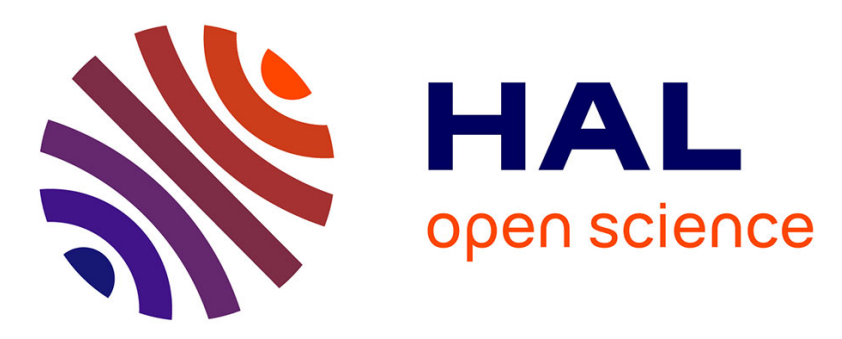

\title{
Density Methods Applied to Form Finding of Initially Stressed Systems
}

\author{
Rene Motro, Bernard Maurin, Nicolas Vassart
}

\section{To cite this version:}

Rene Motro, Bernard Maurin, Nicolas Vassart. Density Methods Applied to Form Finding of Initially Stressed Systems. Michel Frémond; Franco Maceri. Novel Approaches in Civil Engineering, Springer, pp.341-350, 2004, 978-3-540-41836-8. 10.1007/978-3-540-45287-4_30. hal-03342822

\section{HAL Id: hal-03342822 \\ https://hal.science/hal-03342822}

Submitted on 14 Sep 2021

HAL is a multi-disciplinary open access archive for the deposit and dissemination of scientific research documents, whether they are published or not. The documents may come from teaching and research institutions in France or abroad, or from public or private research centers.
L'archive ouverte pluridisciplinaire HAL, est destinée au dépôt et à la diffusion de documents scientifiques de niveau recherche, publiés ou non, émanant des établissements d'enseignement et de recherche français ou étrangers, des laboratoires publics ou privés. 


\section{Density Methods Applied to Form Finding of Initially Stressed Systems}

René Motro, Bernard Maurin and Nicolas Vassart

Laboratoire de Mécanique et Génie Civil, cc 048, Université Montpellier II, F-34095 Montpellier Cedex 5, France

\section{Introduction}

Some contemporary structures like cable nets, membranes and tensegrity systems are initially stressed systems and require form finding processes. The final shape has to fulfil equilibrium conditions. Corresponding relations are not linear and initially a so-called "forcedensity method" has been developed by Linkwitz and Scheck (1971) in order to linearise the problem. This paper describes its utilisation, and an innovative form-finding process the Surface Stress Density Method, based upon the use of isotropic stress tensors, which allows the calculation of broad range of structures and appears as an extension of the existing Force Density Method. Several illustrative examples point out the efficiency of the procedures and also their adaptation to the requirements of either architects or engineers.

\section{Force density method}

This method is used when the system is modelled by an assembly of straight links: cable nets, membranes in case of assimilation to cable nets and tensegrity systems.

\subsection{Equilibrium relation}

Notations The following notations and definitions are used:

$n$, number of nodes, $b$ number of links

$l_{j}^{l i b}$, manufacture length of element " $j$ ", "free" length

$l_{j}^{0}$, Length of element " $j$ " in reference configuration (assembled and not loaded)

$l_{j}$, Length of element " $j$ " in current configuration (assembled and loaded)

Vectors of node coordinates (global reference system) $\{x\}=\left\{x_{1}, \ldots, x_{i}, \ldots, x_{n}\right\}^{t},\{y\}=\left\{y_{1}, \ldots, y_{i}, \ldots, y_{n}\right\}^{t},\{z\}=\left\{z_{1}, \ldots, z_{i}, \ldots, z_{n}\right\}^{t}$ 
Vector of external applied actions $\{f\}=\left\{\ldots, f_{i x}, f_{i y}, f_{i z}, \ldots\right\}^{t}$

External action on node " $i$ ", $\left\{f_{i}\right\}=\left\{f_{i x}, f_{i y}, f_{i z}\right\}^{t}$

Force density coefficient $q_{j}=\frac{T_{j}}{l_{j}^{0}}$ with $T_{j}$, internal effort in element " $j$ ", $T_{j}>0$, traction, $T_{j}<0$, compression.

Equilibrium equation Equilibrium equation for node " $i$ " with force density coefficient is given by:

$$
\begin{aligned}
& \sum_{j \ni i}\left(x_{i}-x_{h}\right) \cdot q_{j}=f_{i x} \\
& \sum_{j \ni i}\left(y_{i}-y_{h}\right) \cdot q_{j}=f_{i y} \\
& \sum_{j \ni i}\left(z_{i}-z_{h}\right) \cdot q_{j}=f_{i z}
\end{aligned}
$$

For the whole system:

$$
[A] \cdot\{q\}=\{f\}
$$

With $[A]$, equilibrium matrix defined by components of members. This matrix has " $3 n$ " lines corresponding to the " $n$ " nodes, and " $b$ " columns, one by element. The number of lines is then reduced to $N, N$ being the number of degrees of freedom.

It is convenient to build this matrix with the connectivity matrix $[C]$, which traduces the relational structure of the system. $[C]$ is a $b$ lines, $n$ columns matrix. General term is $C_{j i}$; for nodes " $i$ " and " $j$ " with no link, $C_{j i}$ is equal to zero. When nodes are linked, then:

$$
C_{j i}=-1 \quad \text { or } \quad C_{j i}=1
$$

Negative value for $i<j$ in the numbering of nodes, and positive value for the opposite case. Each line of equilibrium matrix, corresponding to node " $i$ " and $X$-direction is given by:

$$
[A]_{i x, \bullet}=[C]_{\bullet, i}^{t} \cdot[X]
$$

In this expression $[X]$ is a diagonal matrix comprising the components of the $b$ members along $X$ direction. Similar expressions are derived for $Y$ and $Z$-directions. The whole matrix of equilibrium can be written line by line and organised sequentially according to $X, Y$ and $Z$ directions.

The writing of equilibrium equation of node " $i$ " along $X$-direction in terms of force densities is given by: 
$[C]_{\bullet, i}^{t}[X]\{q\}=\left\{f_{i x}\right\}$

According to diagonal matrix properties, we may express:

$$
[X]\{q\}=[Q]\{X\}
$$

With $[Q]$, diagonal matrix of force densities.

And since:

$$
[X]=[C]\{x\}
$$

Equilibrium equation for all nodes with degree of freedom along $X$ direction, takes the following form:

$$
[C]^{t}[Q][C]\{x\}=\left\{f_{x}\right\}
$$

Values associated with nodes are split in two parts to introduce boundary conditions (for pre stressed systems with fixed nodes). This leads to a partition of the connectivity matrix. The first part is built with terms related to free nodes (subscript "l", and subscript "lx" for $x$-direction), second one with those which qualify fixed nodes along the considered direction (subscript " $f$ " is mentioned for these values, and " $f_{x}$ " for $X$-direction). If, in equations (8), we consider only the $n_{l x}$ equilibrium equations associated with $n_{l x}$ free nodes, we have:

$$
\left[C_{l x}\right]^{t}[Q]\left[C_{l x}\right] x_{l}=f_{l x}-\left[C_{l x}\right]^{t}[Q]\left[C_{f x}\right] x_{f}
$$

We define $\left[D_{x}\right]$ as "connection matrix" containing force density coefficients of nodes which are free along the direction $X$, as follows:

$$
\left[D_{x}\right]=\left[C_{l x}\right]^{t}[Q]\left[C_{l x}\right]
$$

And if we note: 


$$
\left[D_{f x}\right]=\left[C_{l x}\right]^{t}[Q]\left[C_{f x}\right]
$$

We reach the matrix writing of equilibrium with force density method along $X$-direction:

$$
\left[D_{x}\right]\left\{x_{l}\right\}=\left\{f_{l x}\right\}-\left[D_{f x}\right]\left\{x_{f}\right\},
$$

and similar expressions along $Y$ and $Z$-directions.

It is convenient to notice that in case of completely fixed or free nodes, connection matrices are identical for $X, Y$ and $Z$.

Solving system (12) for each direction leads to a geometry satisfying equilibrium equation with chosen force density coefficients.

\subsection{Applications}

Pre and self stressed systems For initially stressed systems, values of force density coefficients are chosen by the designer. These values are stored in matrix $\left[Q^{0}\right]$, and consequently matrices $\left[D_{x}^{0}\right]$ and $\left[D_{x f}^{0}\right]$ are defined

Two cases can be examined:

- pre stressed systems: equilibrium equation $\left[D_{x}^{0}\right] .\left\{x_{l}\right\}=-\left[D_{x f}^{0}\right] .\left\{x_{f}\right\}$

- self stressed systems: equilibrium equation $\left[D_{x}^{0}\right] \cdot\left\{x_{l}\right\}=\{0\}$

Cable nets and membranes Force density method has been developed for cable nets and membranes with an analogy depending on cable net modeling for membranes. Many examples are described in literature. The first studies on tensile membranes were carried out by physical modelling and significant results ensued from F. Otto's works (1973) on soap films. However, these methods are cumbersome and may not provide sufficient accuracy or restrict the variety of possible shapes to minimal area surfaces. Form-finding is today performed with computer-aided numerical procedures. We may firstly distinguish the mechanical approaches based upon a discrete representation of the domain by use of cable networks. This consideration has led to the Force Density Method (Linkwitz and Scheck, 1971). We developed a specific software called Architectural Membranes Design illustrated below according to the different steps of the method 

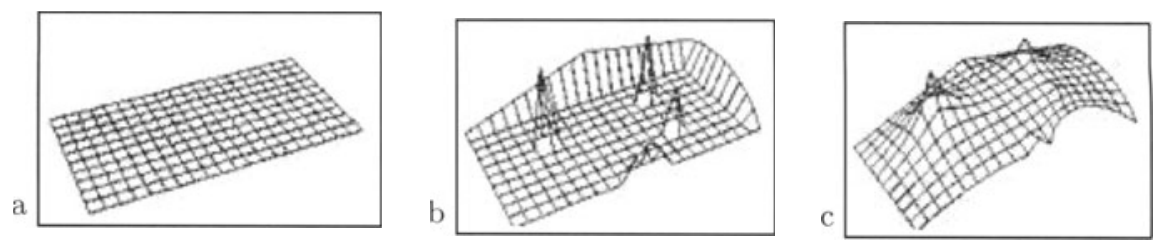

Fig. 1. Formfinding with AMD.

a) initial mesh, b) boundary conditions, c) first shape of Form finding with AMD

Tensegrity systems Tensegrity systems are systems in a stable equilibrium state comprising a discontinuous set of compressed components inside a continuum of tensioned components. It is necessary to emphasise on the fact that matrices for these self stressed systems, are always singular, since for any column or row, the sum of terms is always equal to zero.

$$
\operatorname{rank}\left(\left[D_{x}^{0}\right]\right)=\operatorname{rank}\left(\left[D_{y}^{0}\right]\right)=\operatorname{rank}\left(\left[D_{z}^{0}\right]\right)<n
$$

Consequently equilibrium equations admit an infinity of solutions, since all characteristic determinants vanish when the system to be solved is homogeneous. In case of non specific self stress coefficients, the rank of matrices is generally equal to $n-1$. Solutions are then parametered by only one redundant coordinate, but all the other nodes are confounded with this redundant node. In order to have self stressed geometries, which are not restricted to one point (or one straight line), it is necessary to reduce the connection matrix rank to $n-2$ (respectively $n-3$ ). When this rank is equal to $n-3$, solutions are parametered by three redundant coordinates. The resulting self stressed forms are then planar at best. For planar reticulated systems it is sufficient, but for spatial systems it is necessary to further reduce the rank by one. When the connection matrix rank is reduced to $n-4$, the four redundant nodes which parameter the solutions are then sufficient to generate spatial reticulated self stressed systems.

Example We call "triplex" every tensegrity system comprising six nodes, three struts and nine cables, such as every node is connected to one strut and three cables. In order to find irregular triplexes, we know that it is necessary to reduce the rank of the self stress coefficient connection matrix until $n-4$. Matrices being of dimension 6 , the rank will be equal to 2 . Required self stress coefficients have to be different from zero and to satisfy $q^{0}>0$ for cables and $q^{0}<0$ for struts. All self stress coefficient combinations which lead to a rank equal or less than two can be taken in account.

If, for instance, we choose identical coefficients for members pertaining to a same set of members, that is: 
lower triangle cables:

$$
q_{1}^{0}=q_{2}^{0}=q_{3}^{0}=q_{i}^{0}
$$

bracing cables:

$$
q_{4}^{0}=q_{5}^{0}=q_{6}^{0}=q^{0}
$$

upper triangle cables:

$$
q_{7}^{0}=q_{8}^{0}=q_{9}^{0}=q_{s}^{0}
$$

struts:

$$
q_{10}^{0}=q_{11}^{0}=q_{12}^{0}=-q^{0}
$$

We find after an analytical study (based on Gaussian elimination) that the following relationship has to be satisfied in order to reduce the rank to two:

$$
3 \cdot q_{i}^{0} q_{s}^{0}-\left(q^{0}\right)^{2}=0
$$

Consequently, if $q_{i}^{0}=q_{s}^{0}=1$, then $q^{0}$ can be derived:

$$
q_{i}^{0}=q_{s}^{0}=1 \Rightarrow q^{0}=\sqrt{3}
$$

For every direction, there remain only two independent equilibrium equations. There are then four redundant nodes, which can be located anywhere. Some examples are given on the following figures (in plane views)
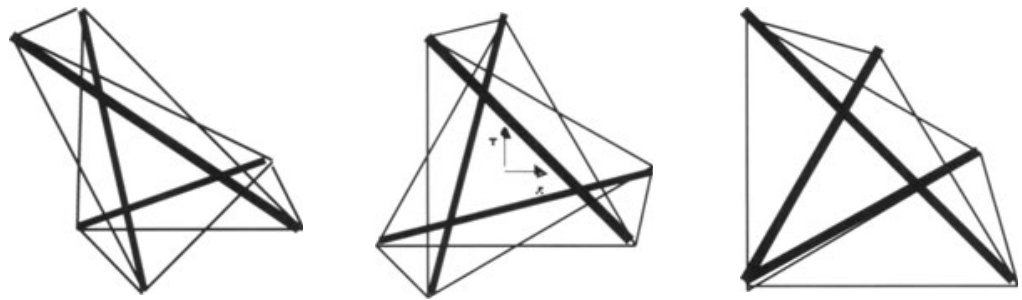

Fig. 2. Different triplex resulting from form finding. Plane views 


\section{Surface stress density method}

\subsection{Introduction}

The stress distribution in a fabric remains an unknown factor even if reference to tensions in cable are done. Most generally, the question is related to the equivalence between a tensile cable net and a pre stressed membrane. Developments point out the inacurracy of this equivalence and therefore the inadequacy of such approach which may lead to significant mistakes. Thus, several methods based on a continuous representation of the fabric with membrane surfacic elements has been proposed. We may quote processes using the Dynamic Relaxation (Barnes, 1975) or Finite Elements methods under large displacement context (Haug and Powell, 1972). They are related to geometrically nonlinear analysis and determinate an equilibrated geometry by deforming a trial configuration whose boundary conditions are modified according to designer's specifications. However, several drawbacks may be put forward. Both the final shape and stresses in the membrane are difficult to control and some areas of the structure may end up in compression. Moreover, these techniques require most of time the use of unfriendly softwares for the architect and high performances hardwares.

\subsection{The Surface Stress Density Method}

This approach is based upon a continuous representation of the domain with triangular membrane surface elements. We note that the mechanical requirement of absence of compressive areas may be satisfied if every elementary Cauchy stress tensor is isotropic, which implies in its local axis $\left(\vec{x}_{m} \vec{y}_{m} \vec{z}_{m}\right)$, that $\left\{\sigma_{0 i s o}^{m}\right\}^{T}=\left\langle\sigma_{0}^{m} \sigma_{0}^{m} 0\right\rangle$ with $\sigma_{0}^{m}>0$. Therefore, the internal forces exerted by the element at its nodes may be written in the global axis of the structure $(\vec{X} \vec{Y} \vec{Z})$ by:

$$
\vec{f} i_{j}^{m}=\frac{-e p_{m}}{2} \sigma_{0}^{m} \ell_{b j} \vec{n}_{j}
$$

where $e p_{m}$ is the fabric thickness and $\vec{n}_{j}$ a normed vector orthogonal to the opposite side of the node. It comes for node 1:

$$
\vec{f} i_{1}^{m}=\frac{e p_{m}}{2} \sigma_{0}^{m} \frac{\ell_{b 1}}{\ell_{h 1}} \overrightarrow{14}=\frac{e p_{m}}{4} \ell_{b 1}^{2} \frac{\sigma_{0}^{m}}{s_{m}} \overrightarrow{14}
$$

with $s_{m}=\frac{1}{2} \ell_{b 1} \ell_{h 1}$ being element area element

By considering the ratio $q_{s m}=\frac{\sigma_{m}^{0}}{s_{m}}$ as the surface stress density for the 

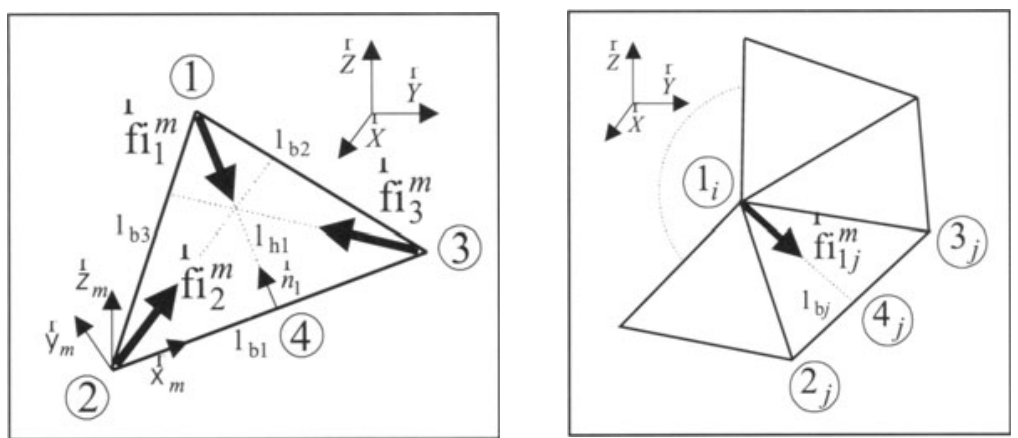

Fig. 3. Membrane element and equilibrium of node

$\vec{f} i_{1}^{m}=\frac{e p_{m}}{4} q_{s} \ell_{b 1}^{2} \overrightarrow{14}$

When $m_{i}$ elements are adjacent to the node $i$ (noted $1_{i}$ on Fig. 3 ), the total internal force is then

$$
\vec{f} i_{j}=\sum_{j=1}^{m_{i}} \vec{f} i_{1 j}^{m}=\frac{e p_{m}}{4} \sum_{j=1}^{m_{i}} q_{s j} \ell_{b j}^{2} \overrightarrow{1_{i} 4_{j}}
$$

With $\left\{\chi_{i}\right\}^{T}=\left\langle X_{1} Y_{1} Z_{1}\right\rangle$, the equilibrium position of node $1_{i}$ verifies the matrix relationship:

$$
\left\{\chi_{i}\right\}=\left[\left[I d_{3}\right]-\left[A_{i}\right]\right]^{-1}\left\{B_{i}\right\}
$$

This equation is the main feature of the Surface Stress Density Method (SSDM)(Maurin and Motro, 1998). It allows to determinate the position of every node in an easy way (the most intricate requirement is a $3 \times 3$ inversion matrix) and to modify it by acting on the $q_{s j}$ values. 
Indeed, $\left[I d_{3}\right]$ is the $3 \times 3$ identity matrix and

$$
\begin{aligned}
{\left[A_{i}\right]=} & \sum_{j=1}^{m_{i}} Q_{s j}\left[\begin{array}{ccc}
X_{3_{j} 2_{j}}^{2} & \text { sym } & \text { sym } \\
X_{3_{j} 2_{j}} Y_{3_{j} 2_{j}} & Y_{3_{j} 2_{j}}^{2} & \text { sym } \\
X_{3_{j} 2_{j}} Z_{3_{j} 2_{j}} & Y_{3_{j} 2_{j}} Z_{3_{j} 2_{j}} & Z_{3_{j} 2_{j}}^{2}
\end{array}\right] \\
\left\{B_{i}\right\}= & \sum_{j=1}^{m_{i}} Q_{s j}\left\{\begin{array}{l}
Y_{2_{j} 3_{j}}\left(X_{3_{j}} Y_{2_{j}}-X_{2_{j}} Y_{3_{j}}\right)+Z_{2_{j} 3_{j}}\left(X_{3_{j}} Z_{2_{j}}-X_{2_{j}} Z_{3_{j}}\right) \\
X_{2_{j} 3_{j}}\left(X_{2_{j}} Y_{3_{j}}-X_{3_{j}} Y_{2_{j}}\right)+Z_{2_{j} 3_{j}}\left(Y_{3_{j}} Z_{2_{j}}-Y_{2_{j}} Z_{3_{j}}\right) \\
X_{2_{j} 3_{j}}\left(X_{2_{j}} Z_{3_{j}}-X_{3_{j}} Z_{2_{j}}\right)+X_{2_{j} 3_{j}}\left(Y_{3_{j}} Z_{2_{j}}-Y_{2_{j}} Z_{3_{j}}\right)
\end{array}\right\}
\end{aligned}
$$

with $\quad q_{s j}=Q_{s j}\left(\sum_{j=1}^{m_{i}} q_{s j} \ell_{b j}^{2}\right)$

Combining with the Force Density Method (FDM) The process allows the management of reinforcing cables located at the edges or above the fabric. If $C_{i}$ cable elements are connected to node $1_{i}$, the total internal force may be expressed as:

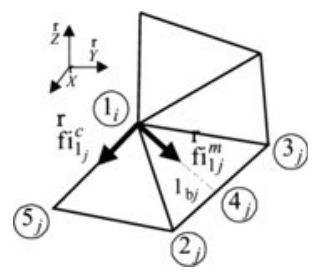

$$
\begin{aligned}
& \vec{f} i_{i}=\sum_{j=1}^{m_{i}} \vec{f} i_{1_{j}}^{m}+\sum_{j=1}^{c_{i}} \vec{f} i_{1_{j}}^{c} \\
& \vec{f} i_{i}=\frac{e p_{m}}{4} \sum_{j=1}^{m_{i}} q_{s j} \ell^{2} \overrightarrow{1_{i} 4_{j}}+\sum_{j=1}^{c_{i}} q_{\ell j} \overrightarrow{1_{i} 5_{j}}
\end{aligned}
$$

Fig. 4. Membrane with cables

\section{Applications}
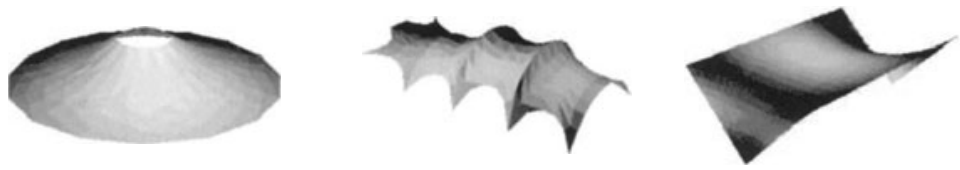

Fig. 5. Some examples of SSDM applications for membranes

When cable elements are located on the edges, their curvature may be managed by changing the ratio between surface coefficients $q_{s j}$ and force density coefficients $q_{l j}$ (Fig.6 a to c). 


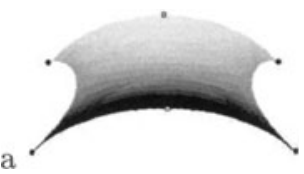

a

Fig. 6. Combination of SSDM and FDM for different values of coefficients

b) $q_{s j}=1$ and $q_{l j}=25$

c) $q_{s j}=1$ and $q_{l j}=100$

\section{Conclusion}

Force density methods appear to be very useful as far as form finding processes are required for initially stressed systems. We contributed to extend the classical force density method to surface stress density method opening also combination possibilities.

\section{References}

1. Barnes M.R.,(1975): Applications of dynamic relaxation to the topological design and analysis of cable, membrane and pneumatic structures. In : W.J. Supple (ed), 2nd International , Conf. on Space Structures, Guildford, pp. 211-219.

2. Haug E., Powell G.H. , (1972): Finite element analysis of nonlinear membrane structures. In : IASS Pacific Symp. Part II on Tension structures and Space Frames, Tokyo and Kyoto, pp 83-92.

3. Linkwitz K., Schek, H.J., (1971): Einige Bemerkungen von vorgespannten Seilnetzkonstruktionen. In : Ingenieur-Archiv 40, Springer-Verlag, pp 145-158.

4. Maurin B. and Motro R., (1998) :The Surface Stress Density method as a Formfinding tool for tensile membrane. In Engineering Structures Vol 20 (8), pp. $712-719$

5. Otto F., (1973) Tensile Structures, Vols 1 and 2. MIT, Cambridge, MA (ed). 